\title{
Hermann Eberhard Fischer zu seinem 50jährigen Doktorjubiläum.
}

Hermann Fischer feiert am 6. März 1905 sein 50jähriges Doktorjubiläum. Er steht im 75. Lebensjahre, und wir können auf ihn mit vollem Rechte dieselben Worte anwenden, mit denen er v. Esmarch zu seinem 80 . Geburtstage begrüßte:

„Ein schönes Alter ist des Lebens Krone;

Nur dem, der sie verdient, wird sie zum Lohne:

Arbeitskräftig und arbeitsfroh, schafft er fleißig und unermüdlich noch heute; alle seine Arbeiten - und ihre Zahl ist wahrlich nicht klein - beweisen, daß er nie geruht, nie stillgestanden hat; sie beweisen alle, daß er bei voller Schätzung und Anerkennung der Leistungen unserer Vorfahren auch jederzeit mit der ihm eigentümlichen Gründlichkeit die neuesten Forschungen verfolgt und sich zu eigen gemacht hat.

Hermann Fischer ist ein Zögling des Friedrich-WillelmsInstitutes, der jetzigen Kaiser-Wilhelms-Akademie für das militärärztliche Bildungswesen.

Hiel promovierte er als

Unterarzt in der Charité im Jahre 1855 mit einer Dissertation: De iritide syphilitica und absolvierte kurz darauf das ärztliche Staatsexamen. Dann war er Assistenzarzt im 3. Artillerie-Regiment und bei der Mobilmachung (1859) Oberarzt in einem Feldlazarett des 3. Armeekorps. Im Jahre $1860 \mathrm{kam}$ er an das Friedrich-Wilhelms. Institut zurück, wurde in demselben Jahre Stabsarzt und zwei Jahre später 'zur Charité kommandiert, wo er $\mathrm{zu}$. erst als Assistent Traubes wirkte. Aus dieser Zeit stammt eine Arbeit über ${ }_{n} B$ emerkenswerte Fälle aus der Klinik desHerrnProf. Traube* in den Annalen des Charité-Krankenhauses. Im dänischen Kriege war er

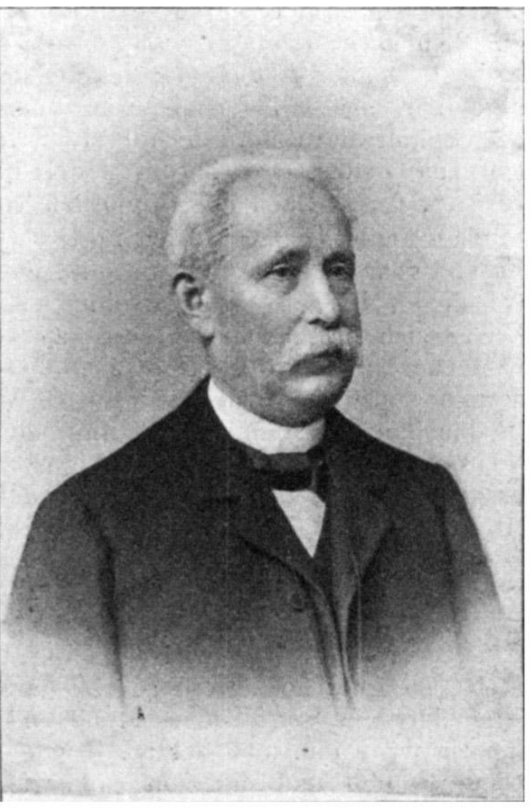

Hermann Eberhard Fischer.

beim 2. schweren Feld-

lazarett in Flensburg, wurde dann Stabsarzt und 1866 Oberstabsarzt im Alexander-Regiment. In Schleswig-Holstein war er mit $\nabla$. Esmarch zusammengetroffen, den er noch im Jahre 1903 als seinen Lehrer in der Kriegschirurgie feierte. Seit Mai 1864 war er außerdem leitender Arzt der "Aeußeren Abteilung", der jetzigen „Chirurgischen Nebenabteilung“ der Charité; damit war er auf sein eigentliches Arbeitsgebiet übergegangen, auf die Chirurgie, der er bis heute treu geblieben ist. Schon sein "Erster Jahresbericht" (1865), der neben einem statistischen 'Teile über Zu- und Abgang etc. eine sehr eingehende Studie über den Hospitalbrand enthält, ist ein Beweis für die Gründlichkeit seines wissenschaftlichen Arbeitens; sie war auch der beste Beweis dafür, daß für die Zeit seiner Tätigkeit die Ansichten Stromeyers und Pirogoffs von dem ngeheimen" Hospitalbrande in der Charité nicht gelten konnten. Aus demselben Jahre stammen noch seine Mitteilungen über das "Krankenzelt", einen leichten Anbau des Sommerlazaretts, und über das "Puerperalfieber in der Charité *. Ein Jahr später habilitierte or sich mit einer Arbeit taber "Die Theorie des Wundfiebers“, und wieder ein Jahr später (1867) erschien das Werk, das ihn wohl am meisten bekannt gemacht hat, seine, Allgemeine Kriegschirurgie “, ein Werk, dem wir an literarischer und sachlicher Forschung und Durchführung auch heute kein besseres gegenüberstellen können. In Pitha-Billroths Handbuch fand es Aufnahme als Abschnitt, Ueber Verletzungendurch Kriegswaffen" und erschien im Jahre 1872 in zweiter Auflage und zugleich als Lieferung $17 \mathrm{a}$ und $\mathrm{b}$ der „Deutschen Chirurgie ${ }^{\alpha}$. 
Als Middeldorpf gestorben war, wurde unser Jubilar am 1. Oktober 1868 als Professor der Chirurgie und Ophthalmologie und Direktor der Chirurgischen Universitätsklinik nach Breslau berufen, wo er in angestrengter und erfolgreicher wissenschaftlicher und praktischer Arbeit bis zum Jahre 1890 , also 22 Jahre lang wirkte. Eine Unterbrechung seiner Lehrtätigkeit brachte auch für ihn, obwohl er seit März 1870 nicht mehr Militärarzt war, der Feldzug 1870/71, und daß er dort, auch unter den schwierigsten Verhältnissen, z. B. vor Metz, seine ganze Arbeitskraft dem Wohle der Verwundeten gewidmet hat, das beweisen unter anderem seine "Kriegschirurgischen Erfahrungen", die im Jahre 1872 erschienen. Das ist wieder ein Buch. ohne dessen Studium, wie die Verfasser der „Kriegschirurgen und Feldärzte" aus eigener Erfahrung bezeugen können, ein Urteil über die chirurgischen Anscliaungen und Leistungen jener Zeit kaum möglich ist. Mit dem Eisernen Kreuze geschmückt kanı er aus dem Kriege zurück. Den Roten Adlerorden vierter Klasse hatte er schon 1864 bekommen, die III. Klasse wurde ihm 1890 und der Kronenorden II. Klasse 1895 verliehen.

Fischer war 1875 Medizinalrat und Mitglied des Medizinalkollegiums und 1885 Geheimer Medizinalrat geworden; eine ganze Reihe von Arbeiten auf dem Gebiete der gerichtlichen Medizin zeigen, da $\beta$ er auch hier auf dem Posten war. Es würde uns zu weit führen, auch nur die Titel aller wissenschaftlichen Arbeiten hier aufzuführen, zu denen er trotz einer riesigen praktischen Tätigkeit inmer noch Zeit fand; ich will nur hervorh eben, daß eine große Zahl dieser und späterer Arbeiten sich eingehend mit den Verletzungen des Zentralnervensystems und ihren Folgen beschäftigt. Sein Lehrbuch der allgemeinen Chirurgie stammt noch aus der Breslauer Zeit (1887); das der speziellen Chirurgie, das einige Jahre später ins Italienische übersetzt wurde, aus dem Jahre 1892. Seiner alten Vorliebe für die Kriegschirurgie verdanken wir noch aus neuester Zeit die ,Kriegschirurgischen Operationen ", den 5. Band der Bibliothek von Coler.

Zum Schlusse möchte ich noch eine Arbeit Fischers nennen die nur von wenigen gekannt und nur von denjenigen anerkannt wird, denen die Schwierigkeit einer solchen Arbeit aus eigener Erfahrung sich bemerkbar gemacht hat - das ist der große Katalog der Büchersammlung der Deutschen Gesellschaft fïr Chirurgie. Das war eine stille, unermüdliche, jahrelange Arbeit, für die ihm allerding, seine ausgedehnte Kenntnis der Literatur zustatten kam. W'ie sehr ihm diese Biblinthek und ihre Vervollkommnung an Ilerzen liegt, beweist die große Zahl der von ihm geschenkten, zum Teil sehr wertvollen und seltenen Werke und die besondere, ebenfalls von ihm überwiesene, nach ihm benannte, fast 1000 Nummeru imfassende "Sammlung H. Fischer".

Wenn ich in dieser kurzen Darstellung auch nur einen kleinen Teil der wissenschaftlichen Leistungen unseres Jubilars nennen konnte, so geht doch wohl so viel darans hervor, daß sein Leben bisher "köstlich war, weil es Mühe und Arbeit gewesen ist" ; kein Wunsch wird ihm bei der 50. Wiederkehr des Tages seiner Promotion so lieb und angenehm sein wie der, daß er noch linge so arbeitsfroh und arbeitskräftig bleiben möge wie an diesem seinen medizinischen Ehrentage.

$$
\text { A. Köhler (Berlin). }
$$

